\title{
MANEJO E PERFIL DE CÃES ATENDIDOS NO HOSPITAL VETERINÁRIO DA UNIVERSIDADE DO OESTE PAULISTA E CARACTERIZAÇÃO DE SEUS PROPRIETÁRIOS.
}

\author{
Laís Costa Muchiutti ; Rosa Maria Barilli Nogueira ${ }^{2}$ \\ ${ }^{1}$ Discente do Curso de Medicina Veterinária, Universidade do Oeste Paulista, Presidente Prudente, SP, Brasil. ${ }^{2}$ Docente da Graduação \\ do Curso de Medicina Veterinária e Mestrado em Ciência Animal, Universidade do Oeste Paulista, Presidente Prudente, SP, Brasil. E- \\ mail: rosa@unoeste.br
}

\section{RESUMO}

Este estudo teve como objetivo verificar o manejo e perfil de cães atendidos no Hospital Veterinário da Universidade do Oeste Paulista e caracterizar seus proprietários. Foi utilizado questionário específico buscando dados dos proprietários como: sexo e idade, e dados dos animais como: raça, idade, sexo, score corporal, alimentação, atividade física, local e frequência de banho, habitat, vermifugação, vacinação e níveis de glicemia. Os resultados foram apresentados na forma descritiva. Conclui-se que os proprietários que frequentam este Hospital Veterinário são na maioria do sexo feminino entre 20 e 39 anos de idade; são atendidos cães, a maioria é sem raça definida de dois meses a dois anos de idade, do sexo feminino, com score corporal ideal do ponto de vista dos proprietários; comem ração seca ou misturada à ração úmida e consomem petiscos; dormem fora de casa; a maioria pratica atividade física todos os dias, tomam banho em casa quinzenalmente, são vacinadas e vermifugadas regularmente e possuem glicemia dentro da normalidade.

Palavras chave: animais; glicose; questionário; manejo.

MANAGEMENT AND PROFILE OF DOGS TREATED AT THE VETERINARY HOSPITAL OF THE UNIVERSITY OF THE WEST PAULISTA AND CHARACTERIZATION OF THEIR OWNERS.

\begin{abstract}
This study aimed to verify the management and profile of dogs treated at the Veterinary Hospital of the University of the West Paulista and characterized their owners. We used questionnaire data from owners seeking as sex and age, and animals as race, age, sex, body score, diet, physical activity, location and frequency of bathing, habitat, worming, vaccination and blood glucose levels. The results were presented in a descriptive way.It is concluded that the owners who attend this Veterinary Hospital are mostly females between 20 and 39 years of age; are treated dogs, most are breed from two months to two years old, female, with ideal body score from the viewpoint of the owners; eat dry food or mixed with moist food and eat snacks, sleep away from home, have physical activity every day, take a bath every two weeks at home, is vaccinated and wormed regularly and have blood glucose within normal limits.
\end{abstract}

Keywords: animals; glucose; questionnaire; management. 


\section{INTRODUÇÃO}

A relação do homem com os animais ainda pouco estudada no Brasil, já mostra uma relação de convivência cada vez mais estreita, o que denota um processo evolutivo onde ser social mostrou-se como vantagem para ambos os lados (FRANCO, 2001).

O cão é o único animal doméstico que teve, radicalmente, todo o seu modo de vida e toda a sua esfera de interesses alterados e hoje é usado para preencher mais necessidades humanas do que qualquer outra espécie doméstica. Talvez por ser preciso tanto de sua companhia e proteção, os cães hoje ocupam posição que muitas vezes transcende seu papel original (BEAVER, 2001).

Segundo Ciampi (2005), depois de adquirir um cão ou gato, diminuem as reclamações do dono sobre pequenos problemas de saúde, e esta relação melhora a sensação de bem-estar, tanto do homem como do animal contribuindo para a socialização (FEITOSA, 2005).

No cenário nacional, a convivência interespécies (do homem com os demais animais) se mostra clara, mediante estimativas populacionais que indicavam em 2001 a existência de 27 milhões de cães e de 11 milhões de gatos como animais de estimação (Associação Nacional dos Fabricantes de Alimentos para Animais - Anfal no ano de 2001). As projeções populacionais brasileiras para 2011 de 34 milhões de cães e 18 milhões de gatos se estendem para outros mascotes, como aves (18 milhões) e peixes ornamentais (8,5 milhões). A propósito, o maior crescimento em 2010 foi registrado para os felinos domésticos com $8 \%$, comparados a $4 \%$ para os canídeos. Essas cifras conferem ao país o segundo lugar no mundo quanto ao número de cães e gatos e o quarto, quando considerada toda a população de pets (ANFAL PET, 2011).

De acordo com a Anfal Pet, $40 \%$ dos animais domésticos consumiam alimento industrializado em 2003. Em 2008, esse percentual subiu para $47 \%$ e em 2010 houve um acréscimo de $4,5 \%$ em relação ao volume obtido no ano precedente.

Os cuidados e atenção não se resumem apenas à alimentação, mas também ao banho, a tosa, roupas, brinquedos $\mathrm{e}$ atendimento veterinário (PHILLIPS-DONALDSON, 2009). Por outro lado, o consumo de alimentos em excesso ou não indicados para os animais, juntamente com o sedentarismo, têm elevado de maneira assustadora a incidência de obesidade, considerada para cães e gatos a afecção nutricional mais comum nas sociedades desenvolvidas. Estima-se que aproximadamente 35 a $40 \%$ dos gatos e 25 a $35 \%$ dos cães apresentem obesidade (GONÇALVES, 2006). Com a obesidade, o risco de outras doenças, como o diabetes, aumentou de forma significativa, havendo necessidade de modificação nutricional, bem como de comportamento, para um tratamento satisfatório (LAFLAMME, 2006; NOGUEIRA; MARCO, 2008; RAMOS, 2011). Desta forma, de acordo com o observado na literatura, este estudo buscou a obtenção do conhecimento sobre o manejo e perfil de cães atendidos no Hospital Veterinário da Universidade do Oeste Paulista, além de caracterizar seus proprietários.

\section{MATERIAL E MÉTODOS}

O estudo foi desenvolvido no setor de Clínica Médica de Pequenos Animais do Hospital Veterinário da Universidade do Oeste Paulista, Presidente Prudente, SP. Fizeram parte do estudo cães atendidos no referido serviço no período de 01/07/09 a 27/12/11, após autorização 
do proprietário através da assinatura do Termo de Consentimento Livre e Esclarecido - TCLE (Anexo 1).

O proprietário respondeu a um questionário semi-estruturado com questões direcionadas a si próprio e ao animal (Anexo 2).

Foi realizada colheita de uma gota de sangue, da região de coxim plantar ou ponta de orelha dos animais, com agulha estéril $25 \times 0,7 \mathrm{~mm}$, a qual foi colocada em glicosímetro portátil $\left(\right.$ Breeze $^{\circledR}$ ou Biocheck ${ }^{\circledR}$ ) para verificação da glicemia, sem jejum prévio no período matutino e vespertino. A análise dos dados foi efetuada recorrendo à estatística descritiva (média, mediana, frequência absoluta e frequência relativa) utilizando o programa Microsoft® Office. Excell 2003.

\section{RESULTADOS E DISCUSSÃO}

No total, foram avaliados 95 animais do atendimento da Clínica Médica de Pequenos Animais do Hospital Veterinário da Universidade do Oeste Paulista após concordância e assinatura do Termo de Consentimento Livre e EsclarecidoTCLE pelo proprietário do animal.

Foi observado desconforto por parte dos proprietários ao responderem a questão sobre "renda financeira mensal", desta forma a mesma foi retirada da avaliação.
Do total dos proprietários atendidos, $65,26 \%$ eram mulheres, e $34,73 \%$ eram do sexo masculino. Quanto à idade dos proprietários, $36,84 \%$ tinham entre dezessete e trinta e nove anos; $28,42 \%$ entre quarenta e cinqüenta e nove anos; 16,84\% tinham idade acima de sessenta anos e $17,89 \%$ optaram por não responder essa questão. O proprietário mais velho era do sexo feminino e possuía 73 anos e o mais jovem tinha 17 anos e também era do sexo feminino.

No estudo de Barbosa (2010) predominou proprietários do sexo feminino, e a idade variou de 26 a 51 anos, resultados semelhantes ao estudo atual.

A raça de maior prevalência foi os sem raça definida (SRD) com 22 animais, seguido do Poodle com 11 animais e em terceiro lugar a raça pinscher representada por 8 animais. As demais raças aparecem na figura 1 . Em estudo realizado por Braga et al. (2010) cães atendidos na Universidade Federal Fluminense, eram na maioria, com raça definida diferente do estudo atual, porém em estudo feito por Barbosa (2010) a maioria dos cães também eram SRD, seguido do poodle e pinscher, resultado idêntico ao atual estudo.

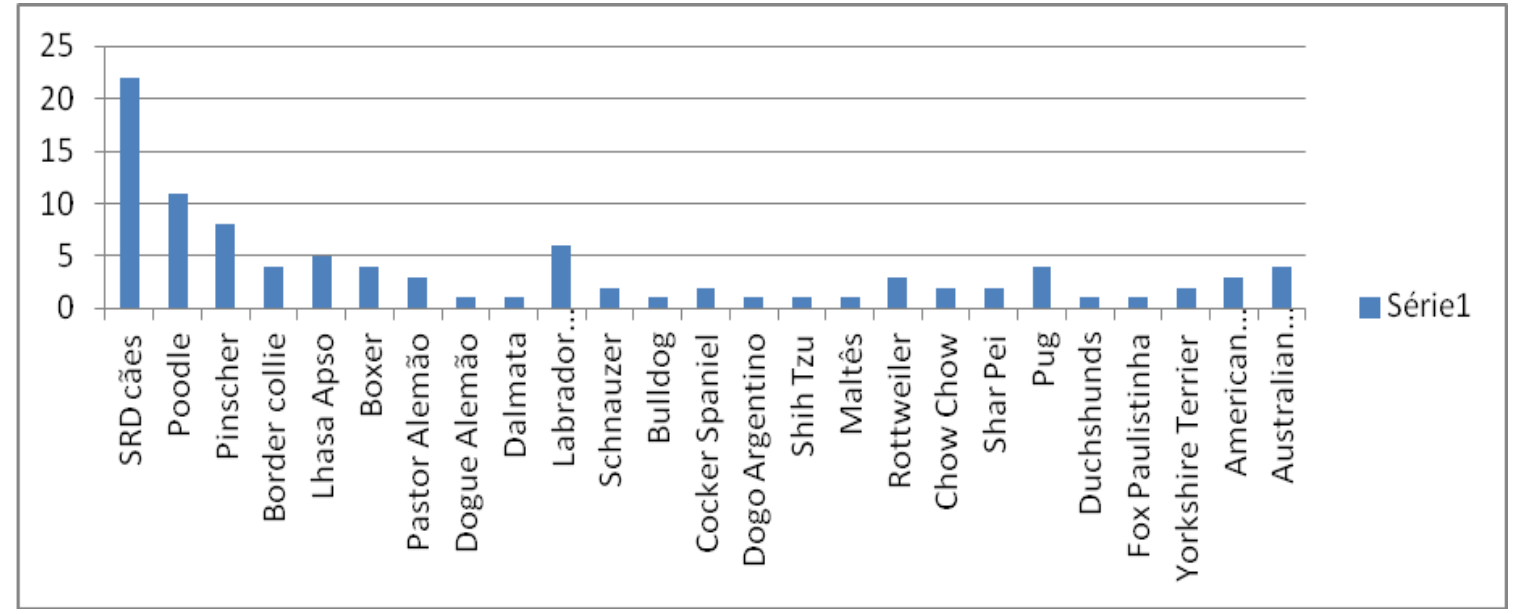

Figura 1. Quantidade de cães e gatos, de acordo com a raça, atendidos no Hospital Veterinário da Universidade do Oeste Paulista. 
A idade predominante dos animais variou entre 2 meses para $34,8 \%$ dos cães; 3 a 5 anos para 20\% dos animais; 6 a 8 anos para 16,8\%; 9 a 11 anos para $13,7 \%$; 12 a 14 anos para $8,4 \%$ e apenas dois animais possuiam idade entre 15-17 anos. Quatro proprietários não souberam informar a idade dos animais. Quanto ao sexo, 64,2\% eram fêmeas, e 35,78\% eram machos.

Em estudo realizado por Braga et al. (2010) na Universidade Federal Fluminense, a maioria dos cães tinham idade entre 1 e 5 anos, seguida sequencialmente por de animais de até 5 meses, de 6 a 10 anos, de 6 meses a 1 ano e a menor porcentagem foi de animais acima de 10 anos de idade. Em outro estudo realizado em Pernambuco por Barbosa (2010), a maioria dos animais atendidos possuía idade de 1,5 a 8 anos. Nos dois estudos acima relatados, foram atendidos mais machos e a idade prevalente foi de 1 a 8 anos, diferindo do estudo atual, onde a maioria dos animais atendidos eram do sexo feminino e com faixa etária entre 2 meses a 2 anos.

No questionamento a respeito do score corporal dos animais, este era avaliado subjetivamente pelos proprietários, classificando seus cães como dentro do peso ideal, magros ou gordos sem que nenhum conhecimento científico sobre esta classificação fosse adotado. Nestes quesitos, $68,42 \%$ dos proprietários responderam achar seu animal dentro do peso ideal; $17,84 \%$ classificaram seus animais como magros e, $13,68 \%$, como gordos.

Quanto ao tipo de alimentação recebida pelo animal, $66,31 \%$ recebiam somente ração seca ou seca misturada a úmida; $9,47 \%$ recebiam somente alimentação caseira e $24,21 \%$ recebiam a mistura do alimento caseiro com a ração seca.

Gonzales et al. (2003) compararam o fornecimento de ração seca, comida caseira e ração seca misturada à comida caseira (mista) e verificaram que animais submetidos à dieta mista (comum no Brasil), tiveram aumento significativo do colesterol e glicemia, o que indica excesso de calorias na dieta, predispondo à obesidade. Para Morooka et al. (2004), o controle de peso em animais é dificultado pelo excesso de alimento oferecido por seus proprietários. Kaiyala et al. (2000) relatam que animais obesos em sobrepeso podem apresentar resistência insulínica (aumentando os níveis de glicemia). Menezes et al. (2005) relataram a caracterização do perfil alimentar de 225 animais, concluindo que, 20\% $(n=45)$ possuíam comportamento alimentar seletivo; $32,44 \%(n=73)$ tinham apetite voraz ou guloso; $6,67 \%(n=15)$ tinham pouco apetite e 40,89\% ( $n=92$ ) possuíam apetite normal, sendo que a maioria dos proprietários forneciam o alimento duas vezes ao dia, seguidos pelos que ofereciam alimento em mais de três vezes ao dia.

$$
\text { Outro dado analisado foi } 0
$$

consumo/oferta de guloseimas (pão, bolacha ou biscoitos), consumo diário em $29,47 \%$ dos animais; consumo em uma frequência de três vezes por semana em $20 \%$; uma vez por semana, em $15,78 \%$, enquanto $34,73 \%$ dos proprietários disseram nunca oferecer esse tipo de alimento aos animais.

O gasto com mimos como roupas e brinquedos se mostrou positiva para $60 \%$, e negativa para $40 \%$ dos entrevistados.

Para o item "regularidade de atividade física" foram propostas quatro variáveis: uma vez por semana, que apresentou freqüência de $22,1 \%$; três vezes por semana, em $16,84 \%$; todos os dias em $37,89 \%$, e $23,15 \%$ dos proprietários disseram que seus animais nunca praticam qualquer tipo de atividade física.

Barbosa (2010) relatou em seu estudo que $94,12 \%$ dos animais possuíam espaço suficiente para se exercitar, sendo que neste estudo, predominou a afirmativa de que os animais se exercitavam em todos os dias da semana. Entretanto, em segundo lugar 
predominou uma gama de animais que nunca se exercitavam, o que gera preocupação, uma vez que o sedentarismo é um fator que, associado à ingestão excessiva de alimentos, leva à obesidade e traz como consequência 0 aparecimento de diversas doenças, e com destaque para o diabetes mellitus.

Quanto ao local aonde os banhos dos cães aconteciam, a casa do proprietário foi o local de maior frequência $(68,42 \%)$, enquanto $31,57 \%$ eram levados ao Pet Shop. Quanto a frequência, $29,47 \%$ recebiam banho semanalmente; quinzenalmente em 34,73\%; mensalmente em $11,57 \%$, e a cada dois meses, ou mais, em $24,21 \%$.

Em relação ao local onde os animais dormiam, 51,57\% dormiam em um lugar fixo fora da residência; $14,73 \%$ sem local fixo e fora da residência dos proprietários; 10,52\% em local fixo, porém dentro da residência; 10,52\% dormiam sem lugar fixo, dentro da residência, e $10,52 \%$ dos cães dormiam no quarto do proprietário. Resende et al. (2005) relataram que dentre 14 animais (12 cães e dois gatos), quatro deles ficavam dentro de casa, quatro ficavam do lado de fora e seis (quatro cães e dois gatos) passavam metade do tempo dentro e outra metade fora de casa.

Em relação à vacinação dos animais, 29,47\% dos proprietários disseram vacinar seus animais somente na campanha de vacinação antirábica promovida pela Prefeitura Municipal de suas respectivas cidades; 55,78\% afirmaram seguir o esquema de vacinação anual com todas as vacinas necessárias e 14,73\% relevaram que não vacinam seus animais.

A vermifugação regular foi positiva para $64,21 \%$ dos animais, enquanto $35,78 \%$ não eram vermifugados. Barbosa (2010) relatou que dos animais de seu estudo, $83,46 \%$ e $83,82 \%$ dos animais eram, respectivamente, vacinados e vermifugados, enquantro $16,54 \%$ e $16,91 \%$ não eram vacinados e vermifugados.

$\mathrm{Na}$ avaliação da glicemia a maior parte dos animais (46,31\%) apresentou valores glicêmicos dentro da normalidade $(70-110 \mathrm{mg} / \mathrm{dL})$ (NOGUEIRA; MARCO, 2008), enquanto 4,21\% apresentaram valores acima da normalidade (>110mg/dL) e 17,89\% ficaram com valores glicêmicos abaixo da normalidade (<70mg/dL). Em 31,57\% não foi possível verificar a glicemia por diversos motivos: comportamento agressivo, doença concomitante sem estabilização como cardiopatas e quadros severos de dermatopatias.

Faria et al. (2005) relataram que em 22 cães onde foi verificada glicemia, 40,90\% apresentavam valores dentro da normalidade e $59,09 \%$ tinham os valores glicêmicos abaixo do limite inferior de referência.

O glicosímetro portátil utilizado para verificação da glicemia mostrou-se de uso fácil, e rápido. Aleixo (2007) relatou em estudo comparativo da mensuração da glicemia em cães utilizando o aparelho portátil, como o deste estudo, e o método de referência laboratorial, que - glicosímetro portátil pode ser considerado clinicamente útil e seguro, uma vez que as diferenças obtidas entre os dois métodos mostraram-se dentro da margem estabelecida pelos órgãos reguladores da área.

Serôdio et al. (2008) relataram, em trabalho comparativo entre a mensuração da glicemia com o glicosímetro digital portátil e o exame laboratorial convencional, que não houve diferença significativa entre os métodos.

O glicosímetro demonstra ser uma ferramenta bastante empregada, por se tratar de um método fácil, de custo relativamente baixo e, rápidos resultados (COHN et al., 2000; WESS; REUSCH, 2000; STEIN; GRECO, 2002), seu uso deve ser incentivado, uma vez que pode culminar em intervenções diagnósticas e terapêuticas precoces (STEIN; GRECO, 2002), devendo-se 
realizar a manutenção periódica desse equipamento.

\section{CONCLUSÃO}

Conclui-se que a relação afetiva entre o homem e cães de estimação tem se estreitado cada vez mais. Pode-se verificar que os proprietários que frequentam o Hospital Veterinário da Universidade do Oeste Paulista são na maioria do sexo feminino, com idade entre 20 e 39 anos; são atendidos cães, sendo a maioria sem raça definida, de dois meses a dois anos de idade, do sexo feminino e com score corporal ideal, do ponto de vista dos proprietários; comem ração seca ou misturada à ração úmida e consomem petiscos; dormem fora de casa; a maioria pratica atividade física todos os dias, tomam banho em pet shop quinzenalmente, são vacinados e vermifugados regularmente $e$ possuem glicemia dentro da normalidade.

\section{AGRADECIMENTOS}

À Pró-Reitora de Pesquisa e Pós-Graduação da Universidade do Oeste Paulista pelo apoio financeiro.

\section{COMITÊ DE ÉTICA}

O estudo foi protocolado (731) e aprovado pela Comissão de Ética no Uso de Animais da Instituição.

\section{REFERÊNCIAS}

ALEIXO, G.A.S. Comparação da glicemia em cães utilizando o glicosímetro portátil e o método de referência laboratorial. Medicina Veterinária, Recife, v.1, n.1, p.89-90, 2007.

ASSOCIAÇÃO NACIONAL DE FABRICANTES DE ALIMENTOS PARA ANIMAIS DE ESTIMAÇÃO - ANFAL Pet. Mercado Pet Brasil. São Paulo: ANFALPET, 2011.

BARBOSA, L.V. Conhecimento sobre bem estar animal pela comunidade acadêmica e acompanhantes de cães e gatos no Hospital Veterinário do departamento de Medicina Veterinária da Universidade Federal Rural de
Pernambuco. 2010. 78f. Dissertação (Mestrado em Ciência Veterinária). Universidade Federal Rural de Pernambuco, Recife.

BEAVER, B.V. Comportamento canino: um guia para veterinários. São Paulo: Roca, 2001.

BRAGA, C.A.; ARRUDA, A.F.O.; CASTRO, M.C.N.; SOARES, A.M.B.; DIECKMANN, A.M. Perfil dos cães e gatos dermatopatas atendidos na Policlínica da Faculdade de Veterinária da UFF. Revista Brasileira de Ciências Veterinária, v.17, n.2, p.73-76, 2010.

CIAMPI, M. Animais de estimação - eles fazem bem à saúde. 2005. Disponível em: http://www.jornalexpress.com.br/noticias/detalhes. php?id_jornal=13950\&id_noticia=212. Acesso em: 28 Ago 2008.

COHN, L.A.; MCCAW, D.L.; TATE, D.J.; JOHNSON, J.C. Assessment of five portable blood glucose meters, a point-of-care analyzer, and color test strips for measuring blood glucose concentration in dogs. Journal of the American Veterinary Medical Association, v. 216, n. 2, p. 198-202, 2000. http://dx.doi.org/10.2460/javma.2000.216.198

FARIA, P.F.; ARAUJO, ARAÚJO, D.F.; SOTOBLANCO, B. Glicemia em cães obesos e senis. Acta Scientiae Veterinariae, v.33, p.47-50, 2005.

FEITOSA, V. Iniciativa pioneira no estado. Diário do Nordeste, 18 Jun 2005. Disponível em: http://diariodonordeste.globo.com/materia.asp?co digo=345258. Acesso em: 27 Ago 2008.

FRANCO, V.L. A relação homem e animal. Revista Digital Isto É, n.341, 2001. Disponível em: http://www.terra.com.br/planetanaweb/341/. Acesso em: 17 Set 2011.

GOLÇALVES, K.N.V. Efeito do tratamento da obesidade sobre a glicemia e insulinemia em gatos. 60f. 2006. Dissertação (Mestrado em Medicina Veterinária) - Faculdade de Ciências Agrárias e Veterinárias, Universidade Estadual Paulista "Júlio de Mesquita Filho", Jaboticabal.

GONZALEZ, F.H.D.; CARVALHO, V.; MOLLER, V.; DUARTE, F.R. Blood bichemical profile in dogs and cats under different feeding diets. Archieves of Veterinary Science, v.8, n.1, p.2327, 2003.

KAIYALA, K.J.; PRIGEON, R.L.; KAHN, S.E.; WOODS, S.C.; SCHWARTZ, M.W. Obesity induced by a High-fat diet is associated with reduced brain insulin transport in dogs. Diabetes, v. 49 p.1525-1533, 2000. http://dx.doi.org/10.2337/diabetes.49.9.1525 
LAFLAMME, D.P. Understanding and managing obesity in dogs and cats. Veterinary Clinics of the North America. Vet North Am Small Animal Practice. v.36, p.1283-1295, 2006. http://dx.doi.org/10.1016/j.cvsm.2006.08.005

MENEZES, D.C.R.; QUESSADA, A.M.; GUIMARÃES, A.L.S. et al. Eutanásia em pequenos animais em Teresina-PI. Semina, v.26, n.4, p.575-579, 2005.

MOROOKA, T.; NIIYAMA, M.; KOUGO, A. et al. Radiographic evaluation of obesity-casused opression of the thoracic cavity in Beagles. Journal of Veterinary Medical Sciences, v.66, n.5, p.489-494, 2004. http://dx.doi.org/10.1292/jvms.66.489

NOGUEIRA, R.M.B.; MARCO, V. Terapêutica das principais endocrinopatias em cães e gatos. In: ANDRADE, S.F. Manual de terapêutica veterinária. São Paulo: Roca, 2008. p.397-431.

PHILLIPS-DONALDSON, D. Rising petfood powers. Petfood Industry, Illinois: Watt Publishing, 2009. p.22-29.

RAMOS, J.M.C. Estudo epidemiológico de Diabetes mellitus na clínica veterinária de Santa Luzia II do Concelho de Guimarães. 2011. 72f. Dissertação (Mestrado Integrado em Medicina Veterinária). Universidade Técnica de Lisboa, Faculdade de Medicina Veterinária, Lisboa.

RESENDE, L.G.; PAIVA, J.B.; ARAÚJO, D.P.; CARVALHO, C.F. Perfil do proprietário de cães e gatos da cidade de Jataí-GO em relação aos cuidados odontológicos de seus animais. $2005 . \quad$ Disponível em: http://www.sbpcnet.org.br/livro/63ra/conpeex/exte nsao-cultura/trabalhos-extensao-cultura/extensaocultura-lara-gisele.pdf. Acesso em: 7 Abr 2011.

SERÔDIO, A.T.; CARVALHO, C.B.; MACHADO, J.A. Glicemia em cães (Canis familiaris) com glucômetro digital portátil e teste laboratorial convencional. Jornal Brasileiro de Ciência Animal, v.1, n.1, p.24-34, 2008.

STEIN, J.E.; GRECO, D.S. Portable blood glucose meters as a means of monitoring blood glucose concentrations in dogs and cats with diabetes mellitus. Clinical Techniques in Small Animal Practice, v.17, n.2, p.70-72, 2002. http://dx.doi.org/10.1053/svms.2002.33041

WESS, G.; REUSCH, C. Evaluation of five portable blood glucose meters for use in dogs. Journal of the American Veterinary Medical Association, v.216, n.2, p.203-209, 2000. http://dx.doi.org/10.2460/javma.2000.216.203 